\title{
Teledermatology Service During the COVID-19 Pandemic in China: A Mobile Application-Based Retrospective Study
}

\author{
Zhanglei Mu' \\ Xiaojing Liu ${ }^{2}$ \\ Kun $\mathrm{Li}^{1}$ \\ Jianzhong Zhang' \\ 'Department of Dermatology, Peking \\ University People's Hospital, Beijing, \\ People's Republic of China; ${ }^{2}$ Department \\ of Dermatology, Shanghai Skin Disease \\ Hospital, Tongji University, Shanghai, \\ People's Republic of China
}

Correspondence: Jianzhong Zhang Department of Dermatology, Peking University People's Hospital,

No. II Xizhimen South St, Beijing, 100044, People's Republic of China Tel +86-10-88325472

Email rmzjz@I26.com
Purpose: During the COVID-19 pandemic, teledermatology service was increased rapidly. The purpose of our study was to analyze the characteristics of patients and common skin diseases via teledermatology during the COVID-19 pandemic in mainland China.

Patients and Methods: During weekends between January 21 and April 4, 2020, the data of patients who used teledermatology service via a mobile application were collected, including gender, age, and diagnosis.

Results: A total of 698 patients (315 men and 383 women), with a mean age of 26 years, used this service. The top ten common diseases in order of proportion were eczema $(22 \%)$, acne $(9 \%)$, atopic dermatitis (9\%), urticaria (5\%), contact dermatitis (5\%), herpes zoster $(3 \%)$, warts $(3 \%)$, folliculitis $(3 \%)$, prurigo $(3 \%)$, and androgenetic alopecia $(2 \%)$. When classified according to age groups, atopic dermatitis was the most common condition for patients in the first decade, acne was more prevalent in the second and third decades, and eczema was the most prevalent condition for all other age groups.

Conclusion: The ten common diseases accounted for the majority of the evaluated cases and varied by age group, allowing individualizing teledermatology services.

Keywords: teledermatology, COVID-19, China, clinical epidemiology, case mix, dermatology

\section{Introduction}

Infection with severe acute respiratory syndrome coronavirus 2 (SARS-CoV-2) was first reported in December 2019 in Wuhan, Hubei province, China. ${ }^{1}$ This virus spread rapidly across the globe. The coronavirus disease 2019 (COVID-19) pandemic has challenged healthcare systems, prompting them to change admission protocols and standardize preventive approaches, including social distancing, hand sanitizing, masks, telemedicine and vaccinations. ${ }^{2-8}$ Although healthcare systems discouraged face-to-face consultations, the demand for dermatological consultations doubled, decreasing patient compliance motivated by misinformation, COVID-19 phobia, and cabin-fever syndrome. ${ }^{8}$

As a result, the COVID-19 pandemic stimulated the development and implementation of telemedicine services. Telemedicine services are available in $88.5 \%$ of counties, cities, and districts of China. ${ }^{9}$ Some telemedicine applications developed by hospitals and companies are certified by the National Health Commission of the People's Republic of China, and consultation fees are covered by health insurance in some cities and commercial medical insurance. 
The teledermatology service requires patients to provide personal information such as gender, age, medical history, and photographs of skin lesions. Subsequently, patients can have online consultations with registered physicians. There are three types of teledermatology services: synchronous, asynchronous, and hybrid. Asynchronous teledermatology uses the store-and-forward technique, and patient information, including photographs, and medical history, are sent online to a physician, who reviews the information and responds minutes or days later. Synchronous (real-time) teledermatology is conducted via video conference. Hybrid teledermatology combines these two technologies. Asynchronous teledermatology is the most widely used; however, there is no direct communication between physicians and patients. Synchronous teledermatology allows physicians to analyze medical records and perform teledermatologic examinations, improving communication. Hybrid teledermatology is less commonly used because it requires a lot of bandwidth and storage space. $^{10}$

Some studies evaluated the demographic and clinical characteristics of patients using teledermatology service in different countries during the COVID-19 pandemic. ${ }^{11-16}$ Several studies were conducted using synchronous teledermatology service in different countries, ${ }^{11,12}$ but not in China. This study reports the experience of an attending physician providing synchronous teledermatology service via the largest telemedicine application in China.

\section{Materials and Methods}

The study was approved by the Ethics Committee of Peking University People's Hospital, and patient consent to review medical records was waived because all patient data were de-identified and securely stored to protect patient confidentiality. The study was conducted in compliance with the Declaration of Helsinki. Haodaifu platform is one of the largest telemedicine providers in China, with approximately 240,000 physicians, and assisted more than 68 million patients until December 2020. It has synchronous, asynchronous, and hybrid teledermatology services. The author (Dr. Mu) was mainly responsible for the synchronous teledermatology service of the entire platform at $6-10 \mathrm{pm}$ every weekend, and there were other physicians responsible for other times. Patients can choose one physician for consultation according to their own schedule. Patients provide personal data, including gender, age, medical history, and photographs of skin lesions via a mobile application and can choose video or phone consultation. The physicians log into the Haodaifu website to check the information and communicate with patients via the smartphone application. Family members, babysitters, or caregivers are responsible for the consultations of children, adolescents, the elderly, and the patients with cognitive or mobility dysfunction. A diagnosis is made based on the information provided during the consultation, and preliminary treatment advice is given. For severe and undetermined conditions, in-person visits to a hospital or clinic are recommended.

Dr. $\mathrm{Mu}$ (male) was in charge of the consultation, and two dermatologists independently reviewed the diagnoses (Dr. Liu and Dr. Li). Demographic data, including age, gender, and diagnosis, were retrieved from the Haodaifu website from January 21 to April 4, 2020, and transferred to an Excel spreadsheet (Microsoft Excel for Mac 16.50). Eczema is defined as non-atopic eczema, including ear eczema, hand eczema, xerotic eczema, nummular eczema, diaper dermatitis, and pruritic dermatitis in the elderly. ${ }^{17}$

Diagnostic information was analyzed and stratified according to gender and age. Statistical analysis was performed using the chi-square test, and p-values of less than 0.05 were considered statistically significant. The data were analyzed using STATA software version 15.1 (Stata Corp, TX) and plotted using Prism version 9 (San Diego, CA).

\section{Results \\ Demographic Characteristics}

A total of 698 patients (315 men and 383 women) made use of this service, of which $55 \%$ of the participants were females. The mean age of the patients was 26 years, with the youngest patient being 24 days old and the oldest patient being 93 years old. The service was mostly used by patients in their third decade of life (25\%), followed by patients in the fourth $(23 \%)$ and the fifth decades of life (23\%) (Figure 1). The use of teledermatology decreased as the age groups increased. In addition, none of our patients were infected with SARS-COV-2.

\section{Disease Characteristics}

The most common skin diseases were eczema (22\%), acne $(9 \%)$, atopic dermatitis $(9 \%)$, urticaria $(5 \%)$, contact dermatitis $(5 \%)$, herpes zoster $(3 \%)$, warts $(3 \%)$, folliculitis $(3 \%)$, prurigo $(3 \%)$, and androgenetic alopecia $(2 \%)$. These ten diseases accounted for $65 \%$ of the evaluated cases, and eczema, acne and atopic dermatitis accounted for $40 \%$ of all diagnoses (Table 1). 


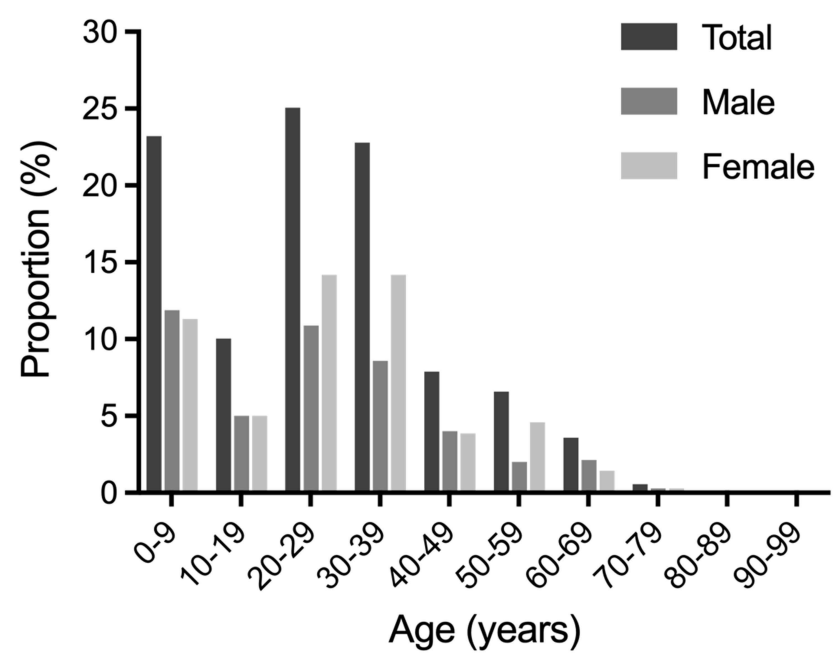

Figure I Gender composition of the different age groups.

\section{Gender Differences}

The total proportion of these ten common diseases was higher in female patients than that in male patients $(68 \%$ versus $61 \%$ ), mainly because of the gender difference in acne (12\% versus $5 \%)$ (Table 2$)$.

\section{Age Differences}

The two most common diseases according to the age group are summarized in Table 3 . The most common disease in the first decade of life was atopic dermatitis

Table I Proportion of Skin Diseases in Patients Using Teledermatology Services

\begin{tabular}{|l|l|}
\hline Diseases & Total (n, \%) \\
\hline Eczema & $153(22)$ \\
Acne & $62(9)$ \\
Atopic dermatitis & $61(9)$ \\
Urticaria & $36(5)$ \\
Contact dermatitis & $34(5)$ \\
Herpes zoster & $23(3)$ \\
Warts & $23(3)$ \\
Folliculitis & $21(3)$ \\
Prurigo & $21(3)$ \\
Androgenetic alopecia & $17(2)$ \\
Tinea pedis & $11(2)$ \\
Seborrheic dermatitis & $10(1)$ \\
Epidermal cyst & $10(1)$ \\
Psoriasis & $9(1)$ \\
Scar/keloid & $8(1)$ \\
Herpes simplex & $7(1)$ \\
Other diseases & $151(22)$ \\
Undetermined diagnosis & $4 I(6)$ \\
\hline
\end{tabular}

Table 2 Proportion of Top Ten Skin Diseases by Gender

\begin{tabular}{|l|l|l|l|}
\hline Diseases & Male (n, \%) & Female (n, \%) & P value \\
\hline Eczema & $67(2 \mathrm{I})$ & $86(22)$ & 0.707 \\
Acne & $16(5)$ & $46(12)$ & $0.00 \mathrm{I}$ \\
Atopic dermatitis & $28(9)$ & $33(9)$ & 0.899 \\
Urticaria & II (3) & $25(7)$ & $0.07 \mathrm{I}$ \\
Contact dermatitis & $14(4)$ & $20(5)$ & 0.635 \\
Herpes zoster & II (3) & $12(3)$ & 0.792 \\
Warts & $13(4)$ & $10(3)$ & 0.264 \\
Folliculitis & $9(3)$ & $12(3)$ & 0.832 \\
Prurigo & $12(4)$ & $9(2)$ & $0.26 \mathrm{I}$ \\
Androgenetic alopecia & $10(3)$ & $7(2)$ & $0.25 \mathrm{I}$ \\
Total & $19 \mathrm{I}(6 \mathrm{I})$ & $260(68)$ & 0.046 \\
\hline
\end{tabular}

$(32 \%)$, acne in the second and third decades $(20 \%$ and $21 \%)$, and eczema in the rest of the age groups $(19 \%$, $30 \%, 28 \%$, and $36 \%$, in the fourth, fifth, sixth and seventh age groups respectively). The second most common disease in each age group was eczema in the first to the third decades, acne in the fourth decade, urticaria in the fifth decade, and heres zoster in the sixth and seventh decades.

\section{Discussion}

The use of teledermatology service increased significantly during the COVID-19 pandemic. A study found that $72.8 \%$ of a cohort could use teledermatology to reduce the risk of COVID-19. ${ }^{18}$ Other studies reported that patients and physicians were interested in using dermatology service via telemedicine. ${ }^{19,20}$

Teledermatology is an important part of telemedicine in China, and many interactive websites and mobile applications allow access to these services. ${ }^{21}$ A survey showed that $18.0 \%$ of physicians offering telemedicine service on the Haodaifu platform in 2017 were dermatologists. ${ }^{22}$ The cost of synchronous teledermatology service is affordable. Taking the Haodaifu application as an example, the consultation fee for new users is one CNY (approximately 0.15 USD), while for regular users it is $29 \mathrm{CNY}$ (approximately 4.5 USD).

Our results indicate that patients aged 20 to 39 years were more likely to utilize teledermatology service, possibly because of the higher use of smartphones. The mean age of patients using these services was lower than that found in previous studies. ${ }^{12-15}$ Women were more likely to use these services than men, consistent with the literature. ${ }^{12-14}$ Patients younger than 10 years were the second largest age group, the proportion in the general population according to the 2019 population data $(23 \%$ 
Table 3 Proportion of the Two Most Common Skin Diseases Between the Ages of 0 and 69

\begin{tabular}{|l|l|l|l|}
\hline Age (Years) & \multicolumn{2}{|l|}{ Diseases (n, \%) } & Total (n, \%) \\
\hline $0-9$ & Atopic dermatitis (52, 32) & Eczema (27, 17) & $79(49)$ \\
$10-19$ & Acne $(14,20)$ & Eczema $(9,13)$ & $23(33)$ \\
$20-29$ & Acne $(36,21)$ & Eczema $(30,17)$ & $66(38)$ \\
$30-39$ & Eczema (31, 19) & Acne (12, 8) & $43(27)$ \\
$40-49$ & Eczema (16, 29) & Urticaria (4, 7) & $20(36)$ \\
$50-59$ & Eczema (13, 28) & Herpes zoster (3, 7) & $16(35)$ \\
$60-69$ & Eczema (9, 36) & Herpes zoster (5, 20) & $14(56)$ \\
\hline
\end{tabular}

versus $11 \%$ ), which may be because their parents or guardians were usually young (aged 20-39 years). The use of teledermatology was lower in patients older than 60 years, and the proportion of elderly patients using the service was lower than that of the elderly people in the population according to the 2019 population data (4\% vs $18 \%$ ), underscoring the need to develop telemedicine applications that are more user-friendly for this population.

The following skin diseases were previously diagnosed via teledermatology: acne, dermatitis, neoplasms, skin infection, papulosquamous disorders, fungal infection/ arthropod bite, and viral diseases in the United States; ${ }^{12}$ psoriasis, atopic dermatitis, hidradenitis suppurativa, and acne in Italy; ${ }^{13}$ nevus, acne, eczema, seborrheic dermatitis, actinic keratosis, and basal cell carcinoma in Spain; ${ }^{14}$ infectious dermatoses, fungal and ectoparasitic infections, papulosquamous disorders, eczema, and dyschromia in India. ${ }^{15}$ Moreover, an internet-based survey showed that the most common dermatological diseases during the COVID-19 pandemic in China were psoriasis $(24.3 \%)$, eczema (19.7\%), dermatitis (12.8\%), vitiligo (11.4\%), acne $(5.3 \%)$, urticaria $(2.5 \%)$, folliculitis $(2.0 \%)$, seborrheic dermatitis $(1.7 \%)$, prurigo $(0.85 \%)$, and tinea pedis $(0.76 \%) .{ }^{16}$ These data showed that acne, eczema, and dermatitis were common in almost all studies, including our study. However, the reported incidence of specific skin conditions via telemedicine varied between different countries. This have been investigated and may be related to factors such as race and geography.

Our results agree with a hospital-based study conducted in Japan, whereby the most common skin diseases were eczema, tinea pedis, urticaria/angioedema, tinea unguium, viral warts, psoriasis, acne, as well as atopic, contact, and seborrheic dermatitis. ${ }^{23}$ The prevalences of common skin diseases in China were androgenetic alopecia $(21.3 \%$ in men and $6.0 \%$ in women), atopic dermatitis $(12.94 \%$ in children aged 1 to 7 years), acne $(8.10 \%)$, herpes zoster (annual incidence of 1.9/1000, and lifetime prevalence of $3.46 \%$ in people aged 50 years and older), psoriasis $(0.47 \%)$, and vitiligo $(0.56 \%) .{ }^{24-30}$ Furthermore, the most common skin diseases in hospitals in China were eczema (19.0-21.8\%) and adult atopic dermatitis $(4.65 \%),{ }^{31-33}$ in line with our results. In addition, the patients in our cohort lived in almost all provincial-level administrative regions (data not shown), and the findings may provide a good reflection on the prevalence of skin diseases in China during the COVID-19 pandemic.

There were age differences in the prevalence of skin diseases. Eczema was predominant in our cohort, especially in the age group 30-69 years, in line with a previous study. ${ }^{23}$ Atopic dermatitis was the most common skin disease in pediatric patients in our cohort, and prevalence was higher than that in Japanese children younger than 10 years and Chinese children aged 1-7 years. ${ }^{23,26}$ Acne ranked first in the age group 10-29 years in our sample and second in Japanese patients aged 16 to 25 years. ${ }^{23}$ Herpes zoster ranked second in the age group 50-69 years in our population and fourth in Japanese patients aged $81-85$ years. ${ }^{23}$ The higher proportion of herpes zoster in this age group may be associated with the presence of other concomitant symptoms, such as neuralgia, stimulating the use of teledermatology service.

Most skin diseases are relatively easy to diagnose based on medical history and clinical examination, facilitating the clinical application of teledermatology service. A previous study showed that asynchronous teledermatology and synchronous teledermatology was preferred in treating acne and complex dermatological conditions, respectively. ${ }^{12}$ According to our experience, synchronous telemedicine is an effective communication tool to dermatologists, although dialects may limit patient-physician communication. Furthermore, the poor quality of mobile video limits the analysis of skin lesions. Thus, the patients should be asked to provide good quality photos prior to the 
video or call to overcome the poor internet quality. In addition, other applications such as WhatsApp have been used in teledermatology. WhatsApp has a large number of users and can deliver synchronous or asynchronous service. However, the limitations of this technology include medical, legal, and ethical issues, privacy, fees, and methods of payment for telemedicine service. ${ }^{34,35}$

\section{Limitations}

This study has limitations. First, although the patients in our cohort presented different skin diseases and came from different regions of China, the data were obtained by one dermatologist using one telemedicine application. Second, age-standardization was not performed, and the results were not compared with face-to-face consultations. Third, the accuracy of diagnosis was not confirmed clinically. In this respect, the diagnostic accuracy of a teledermatology service was less than $90 \%$ and lower than that of in-person services. ${ }^{36}$ Fourth, the sample size was small. Therefore, our findings may not fully reflect the use of teledermatology service by the general population.

\section{Conclusion}

During the COVID-19 pandemic, synchronous teledermatology service was used by many young people. The ten common skin diseases in this study accounted for the majority of all diagnoses, especially eczema, acne and atopic dermatitis. Different age groups have their own common skin diseases, and understanding this helps to provide individualized services. Furthermore, patients should provide high-resolution photographs before video conferences or calls to overcome the poor internet quality. With the increase in mobile phone use and network accessibility, the provision and uptake of teledermatology services are expected to increase in the next decades.

\section{Disclosure}

The authors report no conflicts of interest in this work.

\section{References}

1. Zhu N, Zhang D, Wang W, et al. A Novel Coronavirus from patients with pneumonia in China, 2019. $N$ Engl J Med. 2020;382(8):727-733. doi:10.1056/NEJMoa2001017

2. Gironi LC, Boggio P, Giorgione R, et al. The impact of COVID-19 pandemics on dermatologic surgery: real-life data from the Italian Red-Zone. J Dermatolog Treat. 2020;1-7. doi:10.1080/ 09546634.2020.1789044

3. Pacifico A, Ardigò M, Frascione P, Damiani G, Morrone A. Phototherapeutic approach to dermatology patients during the 2019 coronavirus pandemic: real-life data from the Italian red zone. $\mathrm{Br}$ J Dermatol. 2020;183(2):375-376. doi:10.1111/bjd.19145
4. Cinelli E, Fabbrocini G, Fattore D, Marasca C, Damiani G, Annunziata MC. Safe distance, safe patients! Therapeutic management of oncological patients affected by cutaneous and mucosal adverse events during the COVID-19 pandemic: an Italian experience. Support Care Cancer. 2020;28(9):3991-3993. doi:10.1007/s00520-020-05563-1

5. Bragazzi NL, Mahroum N, Damiani G, Kong JD, Wu J. Effectiveness of community face mask use on COVID-19 epidemiological trends and patterns in Italy: evidence from a "translational" study. Infect Dis (Lond). 2021;53(4):252-254. doi:10.1080/23744235.2021.1883731

6. Cristaudo A, Pigliacelli F, Pacifico A, Damiani G, Iacovelli P, Morrone A. Teledermatology and hygiene practices during the COVID-19 pandemic. Contact Dermatitis. 2020;83(6):536. doi:10.1111/cod.13683

7. Pacifico A, d'Arino A, Pigatto PDM, Malagoli P, Damiani G, Damiani G; Young Dermatologists Italian Network. COVID-19 vaccines do not trigger psoriasis flares in patients with psoriasis treated with apremilast. Clin Exp Dermatol. 2021. doi:10.1111/ced.14723

8. Damiani G, Allocco F, Malagoli P; Young Dermatologists Italian Network. COVID-19 vaccination and patients with psoriasis under biologics: real-life evidence on safety and effectiveness from Italian vaccinated healthcare workers. Clin Exp Dermatol. 2021;46 (6):1106-1108. doi:10.1111/ced.14631

9. National Health Commission of the People's Republic of China; 2021. [Summary of the work of deepening the reform of the medical and health system in 2020]. Available from: http://www.nhc.gov.cn/tigs/ygjb/ 202107/d4bfd518075d4aff9fa3dcd362071b4b.shtml. Accessed August 1, 2021. Chinese.

10. Lee JJ, English JC. Teledermatology: a review and update. Am J Clin Dermatol. 2018;19(2):253-260. doi:10.1007/s40257-017-0317-6

11. McGee JS, Reynolds RV, Olbricht SM. Fighting COVID-19: early teledermatology lessons learned. J Am Acad Dermatol. 2020;83 (4):1224-1225. doi:10.1016/j.jaad.2020.06.027

12. Kazi R, Evankovich MR, Liu R, et al. Utilization of asynchronous and synchronous teledermatology in a large health care system during the COVID-19 pandemic. Telemed J E Health. 2021;27(7):771-777. doi:10.1089/tmj.2020.0299

13. Brunasso AMG, Massone C. Teledermatologic monitoring for chronic cutaneous autoimmune diseases with smartworking during COVID-19 emergency in a tertiary center in Italy. Dermatol Ther. 2020;33(4):e13495. doi:10.1111/dth.13695

14. Sendagorta E, Servera G, Nuño A, Gil R, Pérez-España L, Herranz P. Direct-to-patient teledermatology during COVID-19 lockdown in a health district in Madrid, Spain: the EVIDE-19 pilot study. Actas Dermosifiliogr. 2021;112(4):345-353. doi:10.1016/j.ad.2020.11.020

15. Handa S, Mehta H, Bishnoi A, et al. Teledermatology during the COVID -19 pandemic: experience at a tertiary care centre in North India. Dermatol Ther. 2021;34:e15022. doi:10.1111/dth.15022

16. Li Z, Xu H, Chen J, et al. Internet-based remote consultation facilitates the medical care of patients with chronic skin diseases during COVID-19 pandemic. J Infect. 2021;82(2):e31-e32. doi:10.1016/j. jinf.2020.08.025

17. James WD, Berger TG, Elston DM. Andrews'Diseases of the Skin: Clinical Dermatology. 11th ed. Philadelphia: Sauders; 2011.

18. Temiz SA, Dursun R, Daye M, Ataseven A. Evaluation of dermatology consultations in the era of COVID-19. Dermatol Ther. 2020;33 (5):e13642. doi:10.1111/dth.13642

19. Asabor EN, Bunick CG, Cohen JM, Perkins SH. Patient and physician perspectives on teledermatology at an academic dermatology department amid the COVID-19 pandemic. J Am Acad Dermatol. 2021;84(1):158-161. doi:10.1016/j.jaad.2020.09.029

20. Fluhr JW, Gueguen A, Legoupil D, et al. Teledermatology in times of COVID-19 confinement: comparing patients' and physicians' satisfaction by the standardized brest teledermatology questionnaire. Dermatology. 2021;237(2):191-196. 
21. Zheng Y, Lin Y, Cui Y. Teledermatology in China: history, current status, and the next step. J Investig Dermatol Symp Proc. 2018;19(2): S71-S73. doi:10.1016/j.jisp.2018.09.003

22. Li Y, Yan X, Song X. Provision of paid web-based medical consultation in china: cross-sectional analysis of data from a medical consultation website. J Med Internet Res. 2019;21(6):e12126. doi:10.2196/12126

23. Furue M, Yamazaki S, Jimbow K, et al. Prevalence of dermatological disorders in Japan: a nationwide, cross-sectional, seasonal, multicenter, hospital-based study. $J$ Dermatol. 2011;38(4):310-320. doi:10.1111/j.1346-8138.2011.01209.x

24. Shen Y, Wang T, Zhou C, et al. Prevalence of acne vulgaris in Chinese adolescents and adults: a community-based study of 17,345 subjects in six cities. Acta Derm Venereol. 2012;92(1):40-44. doi:10.2340/00015555-1164

25. Wang TL, Zhou C, Shen YW, et al. Prevalence of androgenetic alopecia in China: a community-based study in six cities. $\mathrm{Br}$ $J$ Dermatol. 2010;162(4):843-847. doi:10.1111/j.1365-2133.20 10.09640.x

26. Guo Y, Li P, Tang J, et al. Prevalence of atopic dermatitis in Chinese children aged 1-7 ys. Sci Rep. 2016;6:29751. doi:10.1038/srep29751

27. Lu L, Suo L, Li J, Pang X. A retrospective survey on herpes zoster disease burden and characteristics in Beijing, China. Hum Vaccin Immunother. 2018;14(11):2632-2635.

28. Zhu Q, Zheng H, Qu H, et al. Epidemiology of herpes zoster among adults aged 50 and above in Guangdong, China. Hum Vaccin Immunother. 2015;11(8):2113-2118. doi:10.1080/21645515.20 15.1016672

29. Ding X, Wang T, Shen Y, et al. Prevalence of psoriasis in China: a population-based study in six cities. Eur J Dermatol. 2012;22 (5):663-667. doi:10.1684/ejd.2012.1802
30. Wang X, Du J, Wang T, et al. Prevalence and clinical profile of vitiligo in China: a community-based study in six cities. Acta Derm Venereol. 2013;93(1):62-65. doi:10.2340/00015555-1397

31. Wang X, Shi XD, Li LF, Zhou P, Shen YW, Song QK. Prevalence and clinical features of adult atopic dermatitis in tertiary hospitals of China. Medicine (Baltimore). 2017;96(11):e6317. doi:10.1097/ MD.0000000000006317

32. Li Q, Yang Y, Chen R, et al. Ambient air pollution, meteorological factors and outpatient visits for eczema in Shanghai, China: a time-series analysis. Int $J$ Environ Res Public Health. 2016;13 (11):1106. doi:10.3390/ijerph13111106

33. Li A, Fan L, Xie L, Ren Y, Li L. Associations between air pollution, climate factors and outpatient visits for eczema in West China Hospital, Chengdu, south-western China: a time series analysis. J Eur Acad Dermatol Venereol. 2018;32(3):486-494. doi:10.1111/ jdv. 14730

34. Lee KJ, Finnane A, Soyer HP. Recent trends in teledermatology and teledermoscopy. Dermatol Pract Concept. 2018;8(3):214-223. doi:10.5826/dpc.0803a013

35. Jakhar D, Kaul S, Kaur I. WhatsApp messenger as a teledermatology tool during coronavirus disease (COVID-19): from bedside to phone-side. Clin Exp Dermatol. 2020;45(6):739-740. doi:10.1111/ ced. 14227

36. O'Connor DM, Jew OS, Perman MJ, Castelo-Soccio LA, Winston FK, McMahon PJ. Diagnostic accuracy of pediatric teledermatology using parent-submitted photographs: a randomized clinical trial. JAMA Dermatol. 2017;153(12):1243-1248. doi:10.1001/ jamadermatol.2017.4280

\section{Publish your work in this journal}

Clinical, Cosmetic and Investigational Dermatology is an international, peer-reviewed, open access, online journal that focuses on the latest clinical and experimental research in all aspects of skin disease and cosmetic interventions. This journal is indexed on CAS.
The manuscript management system is completely online and includes a very quick and fair peer-review system, which is all easy to use. Visit http://www.dovepress.com/testimonials.php to read real quotes from published authors. 